\title{
DESCRIPTION OF ELECTRICAL CHARACTERISTICS OF SEMICONDUCTING COMPOUND-INSULATOR INTERFACE ON THE BASIS OF THE MODEL OF INTERFACE STATES DISTRIBUTED IN ENERGY AND IN SPACE
}

\author{
S. KochowsKI \\ Institute of Physics, Technical University of Silesia \\ ul.Krzywoustego 2, 44-100 Gliwice, Poland
}

\begin{abstract}
The calculation results of the influence of interface state parameters on capacitance-voltage and conductance-voltage characteristics of $n$-GaAs metal insulator semiconductor structures are presented. The $U$-shaped distribution in the energy dimension and Gaussian-type distribution in the space dimension for insulator-semiconductor interface states are assumed.
\end{abstract}

PACS numbers: 72.80.Ey, 73.20.-r, 73.40.Qv

The authors of [1] have suggested that two types of the frequency dispersion of capacitance of the $n$-GaAs metal insulator semiconductor (MIS) structures in the range of voltage corresponding with accumulation structure $[2,3]$ are typical in $n$-GaAs MIS systems and are connected with insulator-semiconductor (I-S) interface.

In this work the results of numerical analysis of influence of I-S interface state parameters on the capacitance-voltage $(\mathrm{C}-\mathrm{V})$ and conductance-voltage $(\mathrm{G}-\mathrm{V})$ characteristics of semiconducting compound MIS structures are presented.

For calculations it is assumed that acceptor and donor interface states exist in the I-S interface. Their density distributions are expressed as follows:

$$
\begin{aligned}
& D_{\mathrm{it}}^{\mathrm{acc}}(E, x)=\left[A /\left(\sqrt{2 \pi} \sigma_{A}\right)\right] \exp \left[a E-x^{2} /\left(2 \sigma_{A}^{2}\right)\right], \\
& D_{\mathrm{it}}^{\mathrm{don}}(E, x)=\left[B /\left(\sqrt{2 \pi} \sigma_{B}\right)\right] \exp \left[-b E-x^{2} /\left(2 \sigma_{B}^{2}\right)\right]
\end{aligned}
$$

for acceptor and donor states, respectively. $E$ is the state energy (in $k T$ units, zero in the semiconductor midgap, $k$ and $T$ have their usual meaning), $x$ is a distance in insulator ( $x=0$ on the semiconductor surface, the state distributions in insulator space are to be considered only). The parameters $a$ and $b$ describe distribution of states in the energy dimension, $\sigma_{A}$ and $\sigma_{B}$ their space distribution, whereas $A$ and $B$ represent the state density for $E=0$ and $x=0$. An influence of the I-S interface states on the capacitance $C_{m}$ and conductance $G_{m}$ of the 
MIS structure is determined by the capacitance and conductance evoked by these states. For their calculations the formulae (3.164a) and (3.164b) from Ref. [4] are used. The additional integration over the insulator thickness is included. In the frame of the assumed model the expressions (2a)-(2d) from [5] for the capture and emission coefficient of carriers by interface states are used. Additionally it is assumed that the electron and hole capture cross-sections $\sigma_{n, p}$ at $x=0$ are energy-dependent: $\sigma_{n, p}(E)=\sigma_{0 n, p} \exp \left(-\alpha_{n, p}|E|\right)$, where $\sigma_{0 n, p}$ are the values in the midgap, $\sigma_{n, p}$ describe the changes of the cross-sections with energy. Using the typical relations $[5,6]$ the characteristics of $C_{m} / C_{0 x}$ and $G_{m} / \omega C_{0 x}$ versus gate voltage $U_{\mathrm{g}}\left(C_{0 x}\right.$ is insulator capacitance, $\left.\omega=2 \pi f\right)$ are calculated for different frequencies $f$ measuring signal. The $n$-GaAs MIS structure $\left(n=3 \times 10^{22} \mathrm{~m}^{-3}\right.$, $C_{0 x}=2.98 \times 10^{-4} \mathrm{~F} \mathrm{~m}^{-2}$ ) is considered. The calculations were performed in the range of $U_{\mathrm{g}}$ corresponding with the accumulation and weak depletion of structure where the peculiarities of the experimental data were observed [3]. At first an influence of parameters of I-S interface on the high-frequency (HF) C-V characteristic has been analysed.

Generally the increasing values of parameters $a, b$ and (or) $A, B$ cause that the change of band bending with $U_{\mathrm{g}}$ is more limited in comparison to the ideal structure. $C_{0 x}$ capacitance is not reached for the realistic voltages $U_{\mathrm{g}}$.
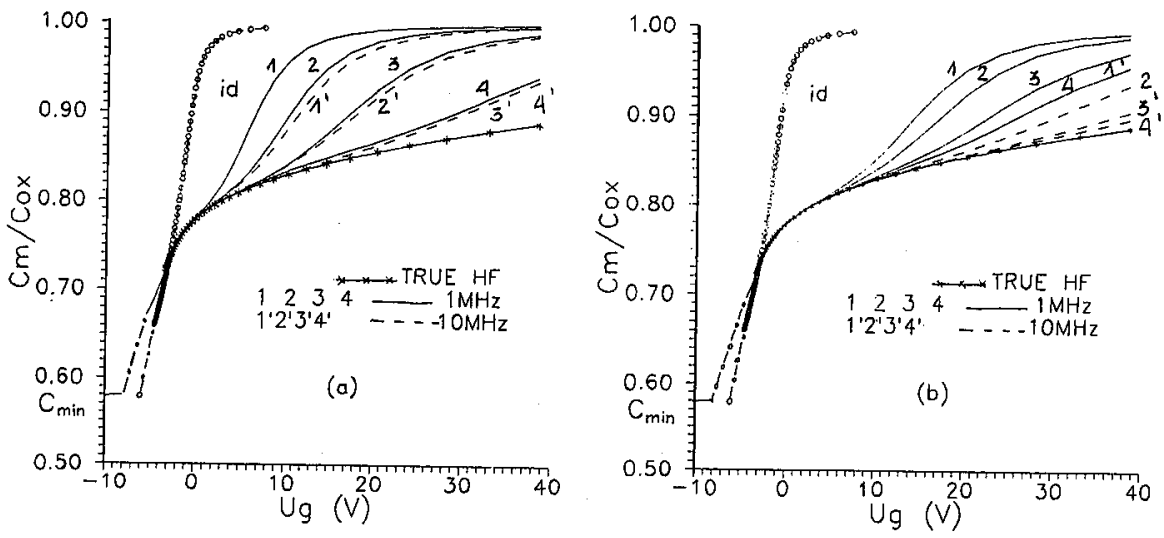

Fig. 1. Influence of $\alpha_{n, p}$ values (a) and $\sigma_{A, B}$ values (b) on capacitance vs. voltage characteristics at $1 \mathrm{MHz}$ and $10 \mathrm{MHz}$ (id - denotes the characteristic of ideal structure). (a) $\alpha_{n, p}=0,0.1,0.2,0.3$ for curves $1-1^{\prime}, 2-2^{\prime}, 3-3^{\prime}, 4-4^{\prime}$, respectively; $\sigma_{A, B}=1 \AA$; (b) $\sigma_{A, B}=0.3,1,3,5 \AA$ for curves $1-1^{\prime}, 2-2^{\prime}, 3-3^{\prime}, 4-4^{\prime}$, respectively; $\alpha_{n, p}=0.2$.

In Figs. 1, 2 and 3 the results of calculations for $A=B=5 \times 10^{15} \mathrm{eV}^{-1} \mathrm{~m}^{-2}$, $a=0.3, b=0.1, \sigma_{0 n}=5 \times 10^{-20} \mathrm{~m}^{2}, \sigma_{0 p}=5 \times 10^{-22} \mathrm{~m}^{2}$ are presented. The curve marked "true HF" is calculated with an assumption that interface states do not follow the measuring signal. From Fig. 1 it results that interface states with the above parameters make a remarkable contribution to the structure capacitance at the frequency $10 \mathrm{MHz}$. The contribution of interface state capacitance depends on the carrier capture cross-sections (Fig. 1a) as well as the space distribution of 

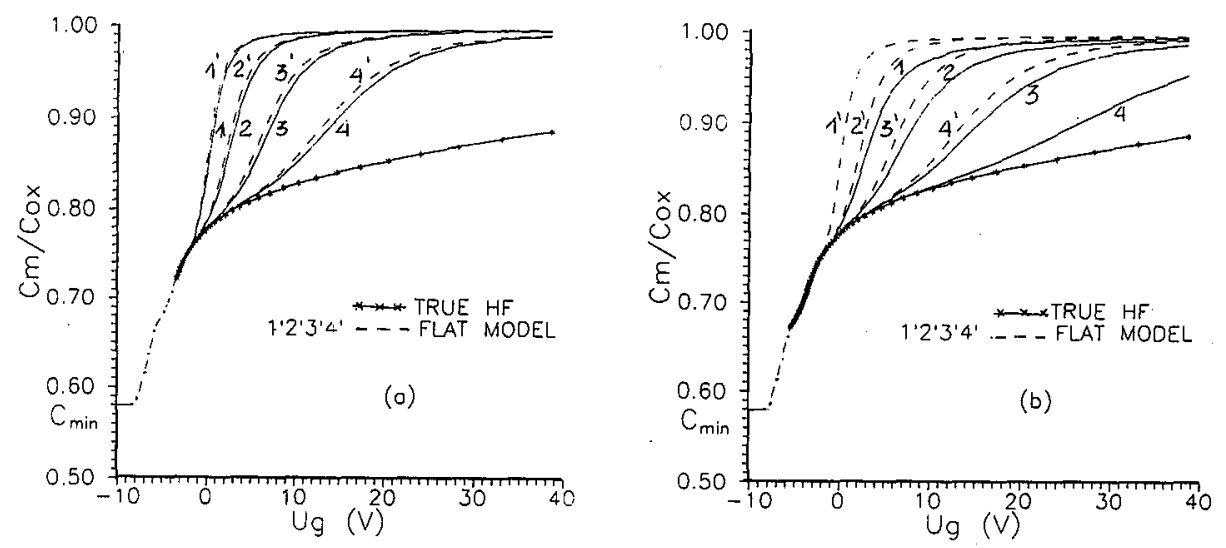

Fig. 2. Influence of $\sigma_{A, B}$ values on capacitance vs. voltage characteristics at various frequencies $\left(f=10^{3}, 10^{4}, 10^{5}, 10^{6} \mathrm{~Hz}\right.$ for curves $1-1^{\prime}, 2-2^{\prime}, 3-3^{\prime}, 4-4^{\prime}$, respectively). (a) $\sigma_{A, B}=0.3 \AA, \alpha_{n, p}=0.2$; (b) $\sigma_{A, B}=5 \AA, \alpha_{n, p}=0.2$.

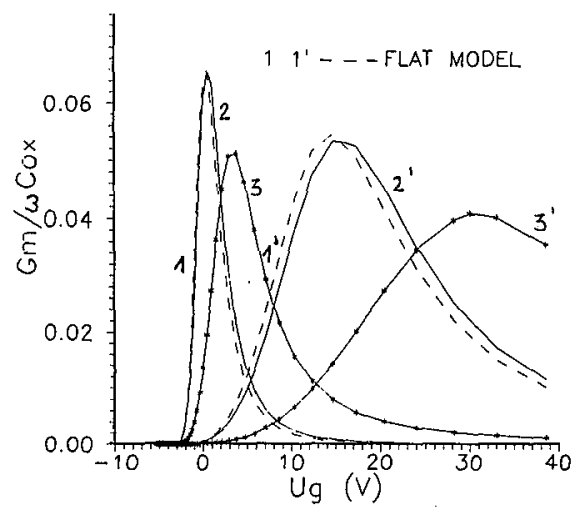

Fig. 3. Plots of conductance vs, voltage characteristics at various frequencies and various $\sigma_{A, B}\left(\sigma_{A, B}=0.3,5 \AA\right.$ for curves $2-2^{\prime}, 3-3^{\prime}$ respectively; $f=10^{3}, 10^{6} \mathrm{~Hz}$ for curves $1-2-3,1^{\prime}-2^{\prime}-3^{\prime}$, respectively).

interface states (Fig. 1b). The influence of space distribution of states is also being revealed in the case of $\mathrm{C}-\mathrm{V}$ and $\mathrm{G}-\mathrm{V}$ curves calculated for different frequencies (Figs. 2 and 3). In Figs. 2a and $2 b$ two types of frequency dispersion of capacitance are shown. Making a comparison between the characteristics calculated for different $\sigma_{A, B}$ and the characteristics calculated for the "flat model" (i.e. with an assumption that the states are localized at the semiconductor surface), it results that changes in the space distribution of interface states can cause the different behaviour of electrical characteristics of MIS structure. The curves presented in Figs. 2 and 3 correspond qualitatively with the experimental $\mathrm{C}-\mathrm{V}$ and $\mathrm{G}-\mathrm{V}$ characteristics, obtained in Ref. [3] for the $n$-GaAs MIS structures produced un- 
der different technological conditions, therefore with different I-S interfaces. The presented results suggest that the interface model (1a) and (1b) can be used to analysis of the I-S interface for semiconducting compounds.

\section{References}

[1] H. Hasegawa, H. Ohno, H. Ishii, T. Haga, Y. Abbe, H. Takahashi, Appl. Surf. Sci. 41/42, 372 (1989).

[2] H. Hasegawa, T. Sawada, Thin Solid Films 103, 119 (1983).

[3] S. Łoś, S. Kochowski, Thin Solid Films 165, 21 (1988).

[4] F. Bertz, in: Surface Physics of Phosphors and Semiconductors, Eds. C.G. Scott, C.E. Reed, Academic Press, London 1975, p. 192.

[5] Li He, H. Hasegawa, T. Sawada, H. Ohno, J. Appl. Phys. 63, 2120 (1988).

[6] W.R. Fahrner, E. Klausmann, D. Brauning, $\mathrm{Si}_{2} / \mathrm{SiO}_{2}$ Intrinsic States and Interface Charges, Chap. 1, Ber. Hahn-Meitner Inst. Kernforsch., Berlin 1984, No 413, p. 33. 